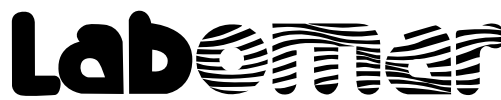

Arquivos de Ciências do Mar

\section{BIODIVERSIDADE DA IGTIOFAUNA NOS CURRAIS DE PESCA NO LITORAL DE ACARAÚ, CEARÁ, BRASIL}

\author{
Ichthyofauna biodiversity in fish-weir in Acaraú Coast, \\ Ceará State, Brazil \\ Toivi Masih Neto', Rodrigo Salles ${ }^{2}$, Emanuel Soares dos Santos ${ }^{3}$, \\ Manuel Alves de Sousa Neto ${ }^{4}$, Luís Parente Maia ${ }^{5}$ \\ ${ }^{1}$ Doutorando do Instituto de Ciências do Mar, Universidade Federal do Ceará, Av. da Abolição 3207,Fortaleza, CE \\ 60165-081. E-mail: toivi@ifce.edu.br \\ 2 Professor do Instituto Federal de Educação, Ciência e Tecnologia do Ceará campus Acaraú. \\ E-mail: rdesalles@hotmail.com \\ ${ }^{3}$ Professor do Instituto Federal de Educação, Ciência e Tecnologia do Ceará campus Acaraú. \\ E-mail: emanuelaqua@yahoo.com.br \\ 4 Técnico em Pesca pelo Instituto Federal de Educação, Ciência e Tecnologia do Ceará campus Acaraú; \\ ${ }^{5}$ Professor do Instituto de Ciências do Mar, Universidade Federal do Ceará, Av. da Abolição 3207, \\ Fortaleza, CE 60165-081. E-mail: parente@ufc.br
}

\begin{abstract}
RESUMO
Esta pesquisa objetivou caracterizar a ictiofauna capturada pelos currais de pesca na Praia de Ilha dos Coqueiros, Acaraú-CE, Brasil, verificar a diversidade, riqueza, equabilidade e abundância das espécies, para conhecer a potencialidade desta arte de pesca para a obtenção do peixes vivos para uso demonstrativo e de educação ambiental em aquários públicos. Foram realizadas 26 coletas entre outubro de 2013 e outubro de 2014 . Foram contabilizados e identificados 1449 peixes capturados em currais de pesca. Os obtidos dados foram utilizados para calcular os índices de diversidade de Shannon-Weaver, Simpson e Berger-Parker, equabilidade de Pielou e riqueza de Margalef. A partir da identificação das espécies foi avaliada a potencialidade para a obtenção de peixes para uso em aquários públicos. Foram capturadas duas classes, 10 ordens, 34 famílias e 60 espécies. As famílias com maior diversidade foram: Carangidae, Haemulidae e Lutjanidae. As espécies com maior frequência em ordem decrescente foram: Haemulon plumierii, Carangoides bartholomaei, Hyporhamphus unifasciatus, Trichiurus lepturus e Diapterus auratus. Todos os índices avaliados mostraram elevada biodiversidade de peixes capturados nos currais de pesca estudados. Várias espécies capturadas são relatadas como utilizadas em aquários públicos, assim esta arte de pesca pode ser usada na obtenção de peixes para este fim.
\end{abstract}

Palavras-chave: Aquário público, Arte de pesca, peixes marinhos, pesca artesanal, peixes vivos.

Recebido em: 25/9/2015

Aprovado em: 15/6/2017

Publicado online em: 20/1/2018 


\section{ABSTRACT}

This research aimed to characterize the ichthyofauna captured by the fish-weirs corrals in the Coconut Island Beach, Acaraú, Ceará State, Brazil, to verify the diversity, richness, equability and abundance of the species, in order to know the potentiality of this fishing gear to obtain live fish for demonstrative use and environmental education in public aquariums. Twenty-six collections were carried out between October 2013 and October 2014. A total of 1449 fish caught in fish-weir were recorded and identified. The data were used to calculate the diversity indexes of Shannon-Weaver, Simpson, Berger-Parker, Pielou equability and Margalef wealth. From the identification of the species was evaluated the potentiality to obtain fish for use in public aquariums. Two classes, 10 orders, 34 families and 60 species were captured. The families with the greatest diversity were: Carangidae, Haemulidae and Lutjanidae. The most frequent species in descending order were: Haemulon plumierii, Carangoides bartholomaei, Hyporhamphus unifasciatus, Trichiurus lepturus and Diapterus auratus. All indices showed high biodiversity of fish caught in the studied fish-weirs. Several captured species are reported as used in public aquariums, so this fishing gear can be used to obtain fish for this purpose.

Key words: Artisanal fishing, fishing gear, live fishes, marine fish, public aquarium.

\section{INTRODUÇÃO}

A pesca e a aquicultura são importantes fontes de alimentação, nutrição, renda e trabalho para centenas de milhões de pessoas em todo o mundo. Em 2014 estas atividades que produziram 167,2 milhões de toneladas, sendo 93,4 milhões de oriundas da pesca, das quais 81,5 milhões de toneladas foram da pesca marinha (FAO, 2016), isto é, esta atividade é responsável pelo fornecimento de aproximadamente $48,7 \%$ de todo pescado produzido no mundo.

Parte da produção da pesca marinha é obtida pelas frotas industriais, mas parte desta é oriunda da pesca artesanal, a qual pode ser definida como aquela em que o pescador, sozinho ou em parceria, participa direta ou indiretamente da captura do pescado, utilizando instrumentos relativamente simples (Ramires et al., 2012).

São diversas as artes de pesca utilizadas pelos pescadores artesanais, entre as quais estão os currais de pesca, também conhecidos como cercos fixos, os quais são encontrados praticamente ao longo de toda costa brasileira, desde o Estado do Paraná até o Estado do Pará, com especial ocorrência na região Nordeste, os quais podem ser encontrados em rios, estuários e praias (Mendonça et al., 2011; Nascimento et al., 2016a).

Um princípio básico para a captura dos peixes utilizando currais de pesca é que estes estejam instalados em locais com influência das marés (Mendonça et al., 2011), na maré alta os peixes entram na armadilha e quando a maré baixa estes ficam presos em seu interior, é neste momento que o pescador realiza a captura com rede auxiliar e transporta a produção obtida em embarcações de pequeno porte movidas a motor ou vela, como canoas ou jangadas.

A costa do Estado do Ceará, Brasil, possui as características necessárias para a instalação dos currais de pesca, sendo muito comum o uso desta tecnologia pelos pescadores 
artesanais da região. A praia de Ilha dos coqueiros, localizada no município de Acaraú, litoral oeste do Ceará, recebe grande influência do estuário do Rio Aracatimirim, sendo parte da atividade pesqueira baseada na exploração dos currais de pesca, tendo como vantagem plataforma continental com grande extensão e pouca inclinação.

Segundo Lima et al. (2013), em levantamento das principais artes de pesca utilizadas nos municípios de Acaraú e Itarema, existem 14 currais em funcionamento, envolvendo 84 pescadores na atividade, tendo como espécies alvo: bonito (Euthynnus alletteratus), serra (Scomberomorus brasiliensis), guarajuba (Carangoides bartholomaei), bejupirá (Rachyncentron canadum), espada (Trichiurus lepturus), galo (Selene sp.), xaréu (Caranx latus), camurim (Tarpon atlanticus), camurupim (Megalops atlanticus), sardinha (Opisthonema oglinum) e agulha (Hyporhamphus unifasciatus). É válido salientar que estão incluídos no citado levantamento os cinco currais de pesca utilizados na presente pesquisa.

É muito importante para o ordenamento pesqueiro conhecer a diversidade dos recursos, assim como o estoque disponível para pesca nas diferentes artes de pesca, desta forma muitos estudos acerca deste assunto vem sendo realizado nos últimos anos no Brasil (Nottingham et al., 2000; Fonteles-Filho \& Espinola, 2001; Piorski et al., 2009; Pinheiro \& Castello, 2010; Lucena et al., 2013; Souza et al., 2015; Lutz et al., 2016), mesmo assim, os estudos existentes ainda são muito aquém do necessário para a manutenção sustentável dos estoques pesqueiros.

Esta pesquisa objetivou caracterizar a ictiofauna capturada pelos currais de pesca na praia de Ilha dos coqueiros, Acaraú-CE, Brasil, verificar a diversidade, riqueza, equabilidade e abundância das espécies, no intuito de conhecer a potencialidade do uso desta arte de pesca para a obtenção do peixes vivos para uso demonstrativo e de educação ambiental em aquários públicos.

\section{MATERIAL E MÉTODOS}

As coletas dos dados da pesquisa foram realizados no porto da praia de Ilha dos

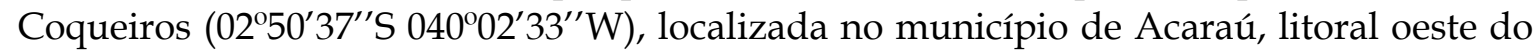
Estado do Ceará, Brasil, distando aproximadamente $220 \mathrm{~km}$ da capital Fortaleza. Este porto recebe os barcos que pescam nos currais de pesca localizados próximo a ele. Na Figura 1 está exposta uma imagem de satélite onde é possível localizar o porto e os currais de pesca amostrados na presente pesquisa.

Na Tabela 1 está exposta a caracterização dos cinco currais de pesca amostrados na presente pesquisa.

Para a realização da coleta dos dados, foram selecionados dias em que a despesca dos currais foi realizada no período da manhã, este manejo teve duração de aproximadamente cinco horas, no qual os pescadores utilizam redes auxiliares para retirada dos peixes que ficam presos dentro dos currais. O pescado obtido era acondicionado em caixas isotérmicas e transportado até o porto local utilizando embarcações de pequeno porte (canoa) com motor de popa de baixa potência.

Durante as despescas rotineiras daquele local, chegando ao porto a produção é contabilizada e dividida, parte é comercializada no próprio local e parte é transportada para venda nos pontos de venda do local ou nos mercados dos municípios vizinhos.

Durante a presente pesquisa, 26 coletas foram realizadas de outubro de 2013 a outubro de 2014, nas quais os peixes capturados, antes de serem divididos e comercializados, 
foram separados e contabilizados segundo a espécie, alguns exemplares foram fotografados com câmera digital, sendo as principais características registradas para que então fosse realizada a identificação com base nos trabalhos de Lessa; Nóbrega (2000), Menezes et al. (2003), Araújo et al. (2004) e Fischer et al. (2011).

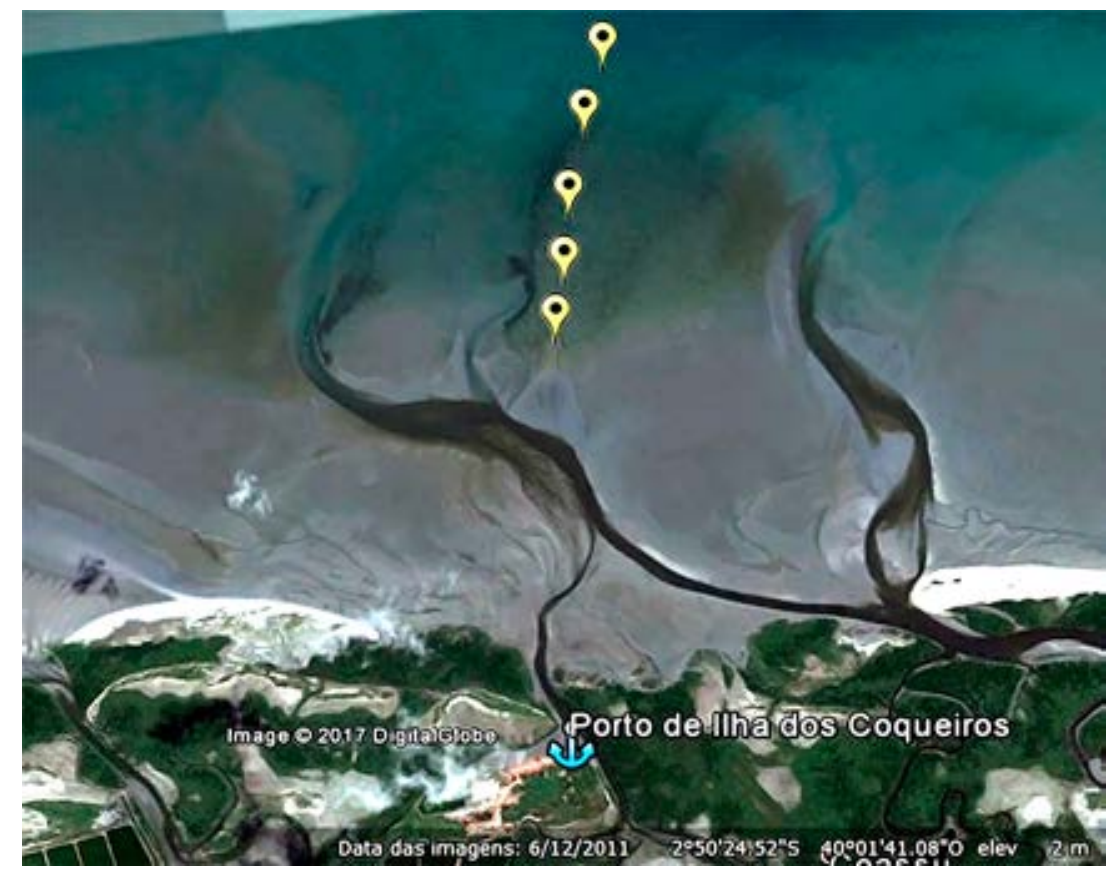

Figura 1 - Localização do porto e dos currais de pesca da praia de Ilha dos coqueiros, Acaraú-CE, Brasil, onde foram realizadas as coletas dos dados de ictiofauna capturada. Fonte: Google Earth (2017). Legenda: O Marcador azul aponta o porto da praia de Ilha dos coqueiros; Os marcadores amarelos indicam a localização dos cinco currais de pesca amostrados.

Tabela 1 - Características dos currais de pesca localizados na praia de Ilha dos coqueiros, Acaraú-CE, Brasil, que foram utilizados para o estudo biodiversidade da ictiofauna capturada.

\begin{tabular}{cccc}
\hline Curral & Tipo de Curral & $\begin{array}{c}\text { Distância* } \\
(\mathbf{k m})\end{array}$ & $\begin{array}{c}\text { Profundidade } \\
(\mathbf{m})\end{array}$ \\
\hline 1 & Terra & 1,791 & 4,0 \\
2 & Terra & 1,962 & 4,0 \\
3 & Terra & 2,165 & 4,0 \\
4 & Meio & 2,836 & 6,0 \\
5 & Fundo & 3,252 & 7,0 \\
* a partir do porto da praia de Ilha dos coqueiros, Acaraú-CE, Brasil.
\end{tabular}

De posse dos dados de abundância de cada espécie (n) e do total de peixes coletados (N) foram realizados cálculos de abundância relativa (Ar) utilizando a Equação 1:

$$
A r=\left(\frac{n}{N}\right) \times 100
$$


Conforme proposto por Paranaguá (1991), segundo o resultado de abundância relativa as espécies podem ser classificadas como:

\begin{tabular}{cc} 
Tabela 2 - Classificação das espécies segundo sua abundancia relativa. \\
\hline Classificação da espécie & Abundância Relativa (\%) \\
\hline Dominante & $\mathrm{Ar}>50 \% ;$ \\
Abundante & $30<\mathrm{Ar} \leq 50 \% ;$ \\
Moderadamente abundante & $10<\mathrm{Ar} \leq 30 \% ;$ \\
Rara & $\mathrm{Ar} \leq 10 \%$. \\
\hline Adaptado de Paranaguá (1991).
\end{tabular}

Conforme sugerido por Magurran (2011) também foram calculados os seguintes índices:

a. Shannon-Weaver $\left(\mathrm{H}^{\prime}\right)$, utilizando a Equação 2:

$\mathrm{H}^{\prime}=-\sum_{i=1}^{S} \frac{\mathrm{n}}{\mathrm{N}} \operatorname{In} \frac{\mathrm{n}}{\mathrm{N}}$

Este indicador fornece resultado em nats.indivíduo ${ }^{-1}$, para facilitar a interpretação da informação obtida é realizada a transformação do resultado para espécie-equivalente, representando o resultado em espécies, utilizando a Equação 3:

$S_{H^{\prime}}=e^{H^{\prime}}$

b. Simpson (S'), conforme a Equação 4:

$$
\mathrm{S}^{\prime}=\Sigma\left(\frac{\mathrm{n}}{\mathrm{N}}\right)^{2}
$$

Da mesma forma, para facilitar a interpretação da informação obtida é realizada a transformação do resultado para espécie-equivalente, utilizando a Equação 5:

$S_{S^{\prime}}=\frac{1}{S^{\prime}}$

c. Berger-Parker (d), conforme a Equação 6:

$d=\frac{n_{\max }}{N}$

Onde: $\mathrm{n}_{\max }$ é o número de indivíduos da espécie mais abundante.

Assim como foi feito para o índice de Simpson, este também será expresso em valor complementar $(1 / \mathrm{d})$.

d. Equabilidade de Pielou (J), a qual foi calculada utilizando a Equação 7:

$J^{\prime}=\frac{H^{\prime}}{H_{\max }}$

Sendo $\mathrm{H}_{\max }$ igual ao $\ln (\mathrm{S})$, onde $\mathrm{S}$ é o número de espécies identificadas.

e. Riqueza de Margalef $\left(\mathrm{d}_{\mathrm{Mg}}\right)$, utilizando a Equação 8:

$d_{M g}=\frac{(S-1)}{\ln N}$ 
A partir dos resultados da identificação das espécies capturadas foi realizada a comparação com dados obtidos em pesquisa bibliográfica nos sites institucionais e materiais de divulgação disponíveis na internet de dois aquários públicos do Brasil, para saber se as espécies utilizadas nestas instituições correspondem aquelas capturadas nos currais de pesca da praia de Ilha dos coqueiros, Acaraú-CE, Brasil.

\section{RESULTADOS E DISCUSSÃO}

Os currais de pesca da praia de Ilha dos coqueiros captura uma grande diversidade de peixes, na identificação dos 1.449 peixes amostrados durante a presente pesquisa, foram observadas duas classes, 10 ordens, 34 famílias e 60 espécies. O detalhamento dos resultados da classificação taxonômica dos peixes capturados nos currais de pesca da praia de Ilha dos coqueiros, Acaraú-CE, Brasil, no período de outubro/2013 a outubro/2014, está exposto na Tabela 3.

Tabela 3 - Classificação taxonômica dos peixes capturados nos currais de pesca da praia de Ilha dos coqueiros, Acaraú-CE, Brasil, no período de outubro/2013 a outubro/2014.

\begin{tabular}{|c|c|c|c|c|c|}
\hline \multirow{2}{*}{ Classe } & \multirow{2}{*}{ Ordem } & \multirow{2}{*}{ Família } & \multirow{2}{*}{ Espécie } & \multicolumn{2}{|c|}{$\begin{array}{c}\text { Total de } \\
\text { indivíduos }\end{array}$} \\
\hline & & & & $\mathbf{n}$ & Ar (\%) \\
\hline \multirow[t]{3}{*}{ Chondrichthyes } & Tetraodontiformes & Ostraciidae & Acanthostracion quadricornis (Linnaeus, 1758) & 7 & 0,48 \\
\hline & & Tetraodontidae & Colomesus psittacus (Bloch \& Schneider, 1801) & 2 & 0,14 \\
\hline & Myliobatiformes & Dasyatidae & Dasyatis guttata (Bloch \& Schneider, 1801) & 7 & 0,48 \\
\hline \multirow[t]{17}{*}{ Actinopterygii } & Pleuronectiformes & Paralichthyidae & Syacium micrurum (Ranzani, 1842) & 1 & 0,07 \\
\hline & Anguilliformes & Muraenidae & Gymnothorax funebris (Ranzani, 1839) & 10 & 0,69 \\
\hline & Beryciformes & Holocentridae & Holocentrus adscensionis (Osbeck, 1765) & 21 & 1,45 \\
\hline & Beloniformes & Belonidae & Ablennes hians (Valenciennes, 1846) & 11 & 0,76 \\
\hline & & Exocoetidae & Hirundichthys affinis (Günther, 1866) & 9 & 0,62 \\
\hline & & Hemiramphidae & Hyporhamphus unifasciatus (Ranzani, 1841) & 109 & 7,52 \\
\hline & Clupeiformes & Clupeidae & Opisthonema oglinum (Lesueur, 1818) & 80 & 5,52 \\
\hline & & Engraulidae & Anchoa spinifer (Valenciennes, 1848) & 3 & 0,21 \\
\hline & & & Anchoa hepsetus (Linnaeus, 1758) & 1 & 0,07 \\
\hline & & & Anchoa filifera (Fowler, 1915) & 1 & 0,07 \\
\hline & Mugiliformes & Mugilidae & Mugil curema (Valenciennes, 1836) & 53 & 3,66 \\
\hline & Perciformes & Acanthuridea & Acanthurus chirurgus (Bloch, 1787) & 10 & 0,69 \\
\hline & & Carangidae & Selene setapinnis (Mitchill, 1815) & 38 & 2,62 \\
\hline & & & Selene vomer (Linnaeus, 1758) & 24 & 1,66 \\
\hline & & & Seriola lalandi (Valenciennes, 1833) & 2 & 0,14 \\
\hline & & & Selar crumenophthalmus (Bloch, 1793) & 4 & 0,28 \\
\hline & & & Trachinotus falcatus (Linnaeus, 1758) & 13 & 0,90 \\
\hline
\end{tabular}




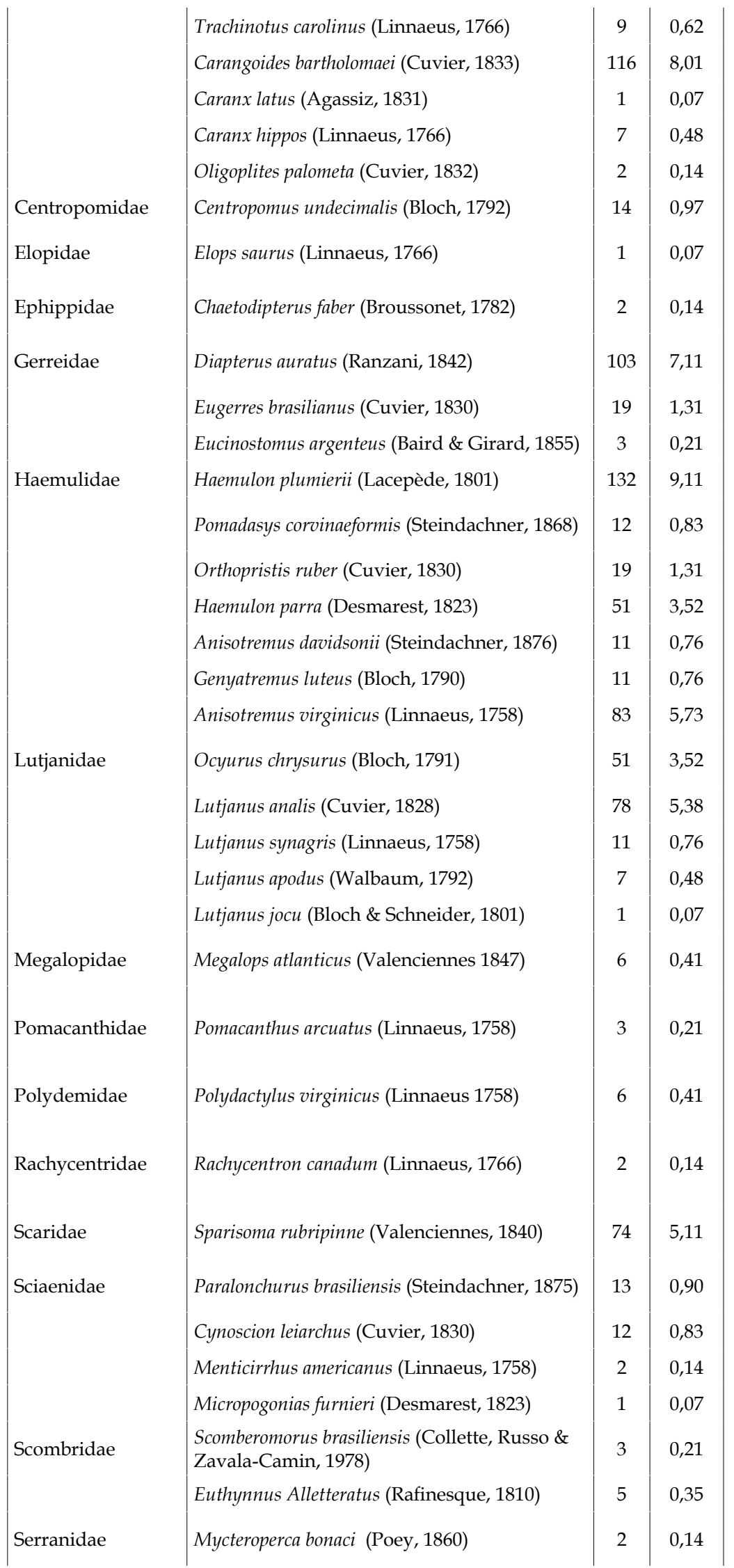




\begin{tabular}{|l|l|l|l|c|c|} 
& & Sphyraeidae & Sphyraena barracuda (Edwards, 1771) & 3 & 0,21 \\
& & Stromateidae & Chloroscombrus chrysurus (Linnaeus, 1766) & 60 & 4,14 \\
& & Peprilus paru (Linnaeus, 1758) & 1 & 0,07 \\
& & Trichiuridae & Trichiurus lepturus (Linnaeus, 1758) & 104 & 7,18 \\
\hline $\mathbf{2}$ & Siluriformes & Ariidae & Arius herzbergii (Bloch, 1794) & 2 & 0,14 \\
\hline
\end{tabular}

Piorski et al. (2009) realizando a identificação da ictiofauna capturada em currais de pesca de dois municípios localizados na ilha de São Luís-MA, Brasil, registraram 57 espécies de peixes distribuídas em 13 ordens e 26 famílias. Observa-se que as quantidades de ordens, famílias e espécies foram bem próximas aquelas alcançadas na presente pesquisa.

A maior diversidade observada neste trabalho foi de peixes da ordem Perciforme, sendo distribuídos em 19 famílias e 45 espécies. Dentre as famílias observadas as que tiveram maior diversidade foram: Carangidae, com 10 espécies; Haemulidae, com 7 espécies; Lutjanidae, com 5 espécies.

Nascimento et al. (2016b) entrevistando os pescadores do município de Cabedelo, situado no litoral norte do estado da Paraíba, Brasil, identificaram as 25 principais espécies de peixes capturadas nos currais de pesca deste local, destas, 15 espécies coincidiram com as que foram relatadas na presente pesquisa, inclusive a família Carangidae também foi a mais relatada por esses autores, das 08 espécies desta família, 05 também foram identificadas na presente pesquisa.

Na Figura 2 pode ser observado o número de espécies de cada uma das 08 famílias com maior representação na ictiofauna capturada nos currais de pesca da praia de Ilha dos coqueiros, Acaraú-CE, Brasil, no período de coleta.

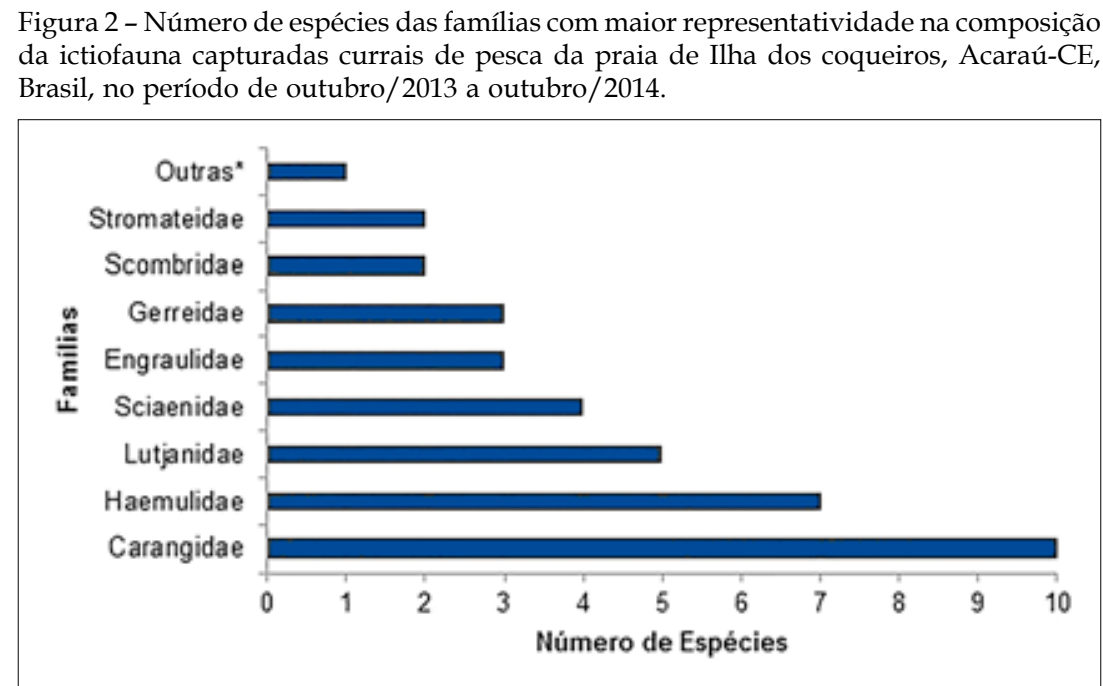

*Outras famílias com apenas uma única espécie capturada.

Entre os 1449 peixes capturados de 60 espécies diferentes, na Figura 3 estão listadas as 15 espécies com maior participação e os respectivos quantitativos de captura, as quais totalizaram 1153 peixes, isto é, 78,9\% do total. 
Figura 3 - Ranking das 15 espécies com maior frequência de captura nos currais de pesca da praia de Ilha dos coqueiros, Acaraú-CE, Brasil, no período de outubro/2013 a outubro/2014.

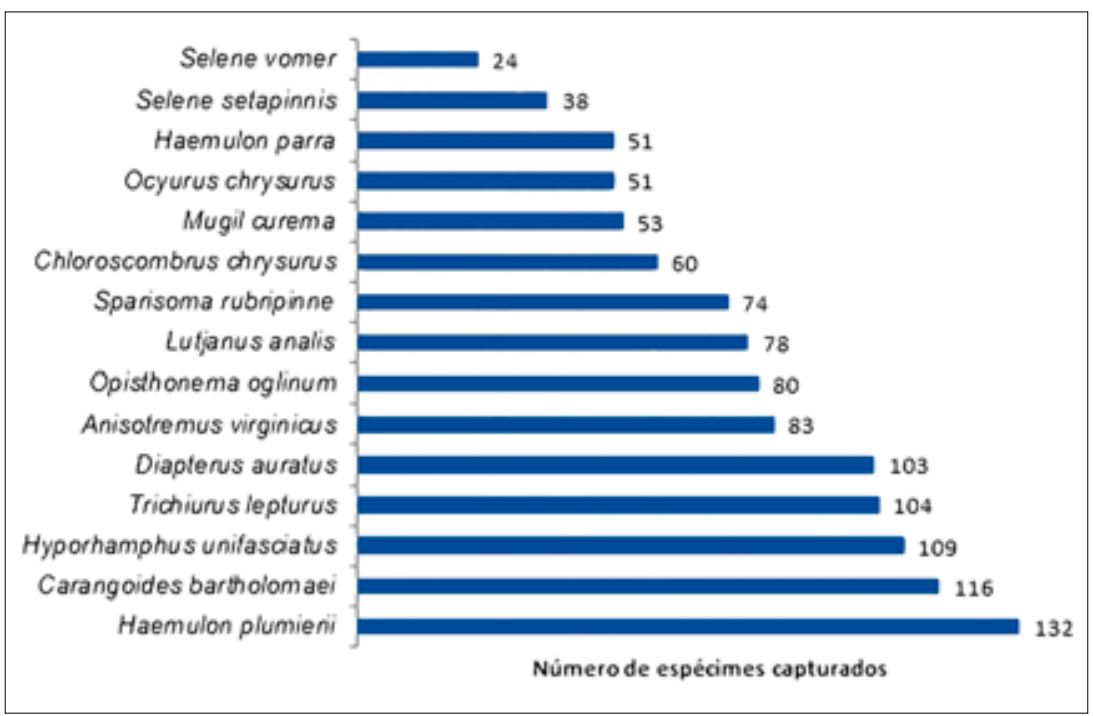

A espécie Haemulon plumierii foi aquela com maior frequência de captura correspondendo a 9,1\% do total (132 peixes), a segunda maior frequência de captura foi de Carangoides bartholomaei, com 8,0\% do total (116 peixes), o terceiro lugar foi de Hyporhamphus unifasciatus (7,5\%, 109 peixes), seguido por Trichiurus lepturus (7,2\%, 104 peixes) e em quinto lugar ficou Diapterus auratus com 7,1\% do total (103 peixes), sendo apenas estas cinco espécies de maior incidência correspondente a 38,9\% de todos os peixes capturados.

Ao verificarmos os valores de abundância relativa (Ar), mesmo as espécies com maior incidência de captura, podem ser classificadas como raras (Ar $\leq 10 \%)$ segundo a escala proposta por Paranaguá (1991). Desta forma, é possível afirmar que é capturada uma grande diversidade de espécies de peixes nos currais de pesca da praia de Ilha dos coqueiros.

Fazendo a relação entre a participação das espécies e suas famílias, temos que, três espécies da família Haemulidae estão entre as que aparecem com maior incidência, Haemulon plumierii, H. parra e Anisotremus virginicus, totalizando 266 indivíduos, sendo a primeira espécie aquela com maior número de peixes capturados nesta pesquisa.

A família Carangidae também foi representada por três espécies, Selene setapinnis, $S$. vomer e Carangoides bartholomaei, totalizando 178 indivíduos capturados, sendo esta última, a segunda maior frequência de captura entre todas as espécies nesta pesquisa.

Na Tabela 4 estão os resultados dos índices de diversidade utilizados na avaliação da biodiversidade de peixes capturados nos currais de pesca da praia de Ilha dos coqueiros, Acaraú-CE, Brasil, onde em todos os indicadores utilizados foi apontada biodiversidade elevada.

Tabela 4 - Resultados dos índices de diversidade utilizados para avaliar biodiversidade capturada pelos currais de pesca da praia de Ilha dos coqueiros, Acaraú-CE, Brasil, no período de outubro/2013 a outubro/2014.

\begin{tabular}{lcccc}
\hline Índices de Diversidade & Sigla & Resultado & Sigla & Resultado \\
\hline Shannon-Weaver & $\mathrm{H}^{\prime}$ & 3,3 & $\mathrm{~S}_{\mathrm{H}^{\prime}}$ & 27,1 \\
Simpson & $\mathrm{S}^{\prime}$ & 0,05 & $\mathrm{~S}_{\mathrm{s}^{\prime}}$ & 19,83 \\
Berger-Parker & $\mathrm{d}$ & 0,09 & $\mathrm{~S}_{\mathrm{d}}$ & 10,98 \\
Equabilidade de Pielou & $\mathrm{J}$ & 0,81 & n.a. & n.a. \\
Riqueza de Margalef & $\mathrm{d}_{\mathrm{Mg}}$ & 8,1 & n.a. & n.a. \\
\hline
\end{tabular}

S: valor em espécie equivalente para seu índice correspondente; n.a.: não se aplica. 
Quanto maior o valor do resultado do índice de Shannon-Weaver $\left(\mathrm{H}^{\prime}\right)$ maior é a biodiversidade (Scolforo et al., 2013), devendo o resultado estar no intervalo de 1,5 a 3,5 (Lima et al., 2016). O valor obtido foi de 3,3 nats.indivíduo ${ }^{-1}$, bem próximo ao limite do intervalo de referência, indicando assim a elevada diversidade de espécie de peixes capturada pelos currais de pesca da praia de Ilha dos coqueiros. Convertendo o resultado em espécie equivalente este passa a ser 27,1 espécies.

O valor calculado do índice de Simpson $\left(S^{\prime}\right)$ ocorre na escala de zero a um, sendo que os valores próximos de 1,0 indicam menor diversidade (Scolforo et al. 2013; Lima et al., 2016). Desta forma, o valor de $S^{\prime}$ igual a 0,05 indica a grande biodiversidade obtida na presente pesquisa, sendo 19,83 o resultado em espécie equivalente.

O valor do índice de Berger-Parker (d) decresce concomitantemente com o crescimento da riqueza de espécies (Magurran, 2011), desta forma o valor de "d" igual a 0,09, indica biodiversidade alta de peixes capturados nos currais de peixes da praia de Ilha dos coqueiros, Acaraú-CE.

A equabilidade de Pielou (J) analisa a distribuição dos indivíduos entre as espécies na amostra, para este índice o resultado foi 0,81, segundo Odum (2010), os resultados variam de zero, para a diversidade mínima, até um, quando a diversidade é máxima, os valores superiores a 0,50 são considerados como significantes, desta forma os resultados obtidos na presente pesquisa mostram equabilidade significante entre as espécies de peixes encontradas.

Em relação ao índice de riqueza de Margalef $\left(\mathrm{d}_{\mathrm{Mg}}\right)$ foi obtido o valor 8,1, o qual indica grande biodiversidade. Conforme Margalef (1958), valores inferiores a 2,0 apontam baixa diversidade (em geral em resultado de efeitos antropogênicos) e valores superiores a 5,0 são considerados como indicador de grande biodiversidade.

Ao comparar as espécies capturadas, com informações dos aquários públicos no Brasil disponíveis nos sites institucionais, em materiais de divulgação e nas visitas aos aquários públicos marinhos, é possível constatar que algumas das que foram capturadas correspondem as mesmas utilizadas nestas instituições.

Como exemplo, é possível citar as espécies observadas no Aquário de São Paulo (Novembro de 2016): Gymnothorax funebris e Megalops atlanticus, ambas tiveram exemplares capturados nesta pesquisa; outras como a raia-prego Dasyatis americana, que, nesta pesquisa, foi capturada outra espécie da mesma família, a Dasyatis guttata; e o baiacu cara de cachorro Arothron nigropunctatus, tendo como espécie substituta que foi capturada o baiacu cofre Acanthostracion quadricornis.

Já o Aquário de Santos (Viva Santos, 2017) declara, entre outros peixes, expor robalos e caranhas, ambos tiveram exemplares capturados nos currais de pesca estudados. O robalo flecha Centropomus undecimalis teve exemplares capturados; e as caranhas, que é o nome popular associado a algumas espécies de Lutjanídeos, normalmente o Lutjanus jocu, L. apodus ou L. griseus, destas três espécies as duas primeiras também foram capturadas.

Além destas espécies outras são potencialmente exploráveis dentre as quais é válido citar: a guaiúba Ocyurus chrysurus, o bijupirá Rachycentron canadum, barracuda Sphyraena barracuda, galo Selene vomer, e a arabaiana ou olhete Seriola lalandi.

Todas estas espécies acima citadas foram capturadas nos currais de pesca da Ilha dos Coqueiros, desta forma esta arte de pesca pode ser potencialmente usada para captura de peixes para uso em aquário públicos, onde é importante salientar que este tipo de armadilha mantém os animais vivos e em boas condições até o momento da despesca do curral, 
sendo necessário desenvolver técnicas de captura, anestesia, transporte e aclimatação destas espécies para o uso desejado.

\section{CONCLUSÃO}

Os currais de pesca da praia de Ilha dos coqueiros capturam uma grande diversidade de peixes, a saber: duas classes, 10 ordens, 34 famílias e 60 espécies. Dentre as famílias observadas as que tiveram maior diversidade foram: Carangidae, com 10 espécies; Haemulidae, com 7 espécies; Lutjanidae, com 5 espécies.

A espécie Haemulon plumierii foi aquela com maior frequência de captura seguida pela Carangoides bartholomaei, esta pela Hyporhamphus unifasciatus, depois a Trichiurus lepturus e em quinto lugar ficou Diapterus auratus essas cinco foram responsáveis por 38,9\% de todos os peixes capturados

Em todos os índices utilizados a avaliação da biodiversidade de peixes capturados nos currais de pesca da praia de Ilha dos coqueiros foi considerada elevada.

Várias das espécies capturadas são relatadas como utilizadas em aquários públicos, desta forma esta arte de pesca pode ser potencialmente usada para captura de peixes para este fim, considerando que os animais ficam vivos e em boas condições até o momento da despesca do curral.

\section{REFERÊNCIAS BIBLIOGRÁFICAS}

Aquário de São Paulo (2017). O Site instituconal do Aquário de São Paulo - O aquário do tamanho de São Paulo, Link animais do aquário de São Paulo. Disponível em: http:/ / www.aquariodesp.com.br/novo/visitas/animais. Acessado em: 09 de junho de 2017.

Araújo, M.E.; Teixeira, J.M.C. \& Oliveira, A.M.E. Peixes Estuarinos Marinhos do Nordeste Brasileiro: guia ilustrado. Fortaleza: Edições UFC, 2004.

FAO - Food and Agriculture Organization. The Stateof World Fisheries and Aquaculture 2016. Roma: FAO, 2016. Disponível em: http://www.fao.org/3/a-i5555e.pdf Acessado em: 27 abr. 2017.

Fischer, L.G.; Pereira, L.E.D. \& Vieira, J.P. Peixes estuarinos e costeiros. 2 ed., Rio Grande, 2011. 131 p.

Fonteles-Filho, A. A. \& Espínola, M. F. A. Produção de pescado e relações interespecíficas na biocenose capturada por currais-de-pesca, no Estado do Ceará. Ceará, Bol. Téc. Cient. CEPNOR, Belém, v. 1, n. 1, p. 111 - 124, 2001.

Lessa, R. \& Nóbrega, M.F. Guia de Identificação de Peixes Marinhos da Região Nordeste. Programa REVIZEE/SCORE-NE. Recife, DIMAR, 2000, 138 p.

Lima, E.H.S.M.; Melo, M.T.D.; Silveira, F.; Betti, H.T.; Zmyslowski, C.T. \& Silva, H.A.B. Levantamento das principais artes de pesca utilizadas nas comunidades pesqueiras na área de atuação do projeto TAMAR-ICMBIO, Regional Ceará, 2013, 47 p. Disponível em: http:/ / tamar. org.br/arquivos/ARTES-PESCA-CEARA_Levantamento.pdf. Acessado em: 31 de maio de 2017.

Lima, M.S.C.S.; Souza, C.A.S. \& Pederassi, J.S. Qual Índice de Diversidade Usar? Cadernos UniFOA, Volta Redonda, n. 30, p. 129-138, abr. 2016.

Lucena, F.P.; Cabral, E.; Santos, M.C.F.; Oliveira, V.S. \& Bezerra, T.R.Q. A pesca de currais para peixes no litoral de Pernambuco. Bol. Téc. Cient. CEPENE, v. 19, n. 1, p. 93-102, 2013. 
Lutz, I.A.F.; Lima, W.M.G.; Gonçalves-Filho, I.A.; Cintra, I.H.A. \& Silva, B.B. Fishery production landing in a northern Brazilian estuary (Bragança, Pará). Acta Fish. Aquat. Res., v. 4, n. 2, p. 125-136, 2016. http://dx.doi.org/10.2312/ActaFish.2016.4.2.125-136.

Magurran, A. E. Medindo a diversidade biológica. Paraná: UFPR, Brasil, 2011, 256 p.

Margalef, R. Information theory in Ecology. Int. Journal Gen. Sys. v. 3, p. 36-71, 1958.

Mendonça, J.T.; Machado, I.C.; Jensen, L.V.; Campolimi, M.B.; Lucena, A. \& Cardoso, T.A. Management of the fish-weir fisheries at the Cananéia-Iguape-Ilha Comprida estuary. Arq. Ciên. Mar, v. 44, n. 2, p. 36 - 51, 2011.

Menezes, N.A.; Buckup, P.A.; Figueiredo, J.L. \& Moura, R.L. Catálogo das Espécies de Peixes Marinhos do Brasil. São Paulo: Museu de Zoologia USP, 2003. 164p.

Nascimento, G.C.C.; Córdula, E.B.L.; Lucena, R.F.P.; Rosa, R.S. \& Mourão, J.S. Characterization of artisanal fishing in fishweirs, the north coast of Paraiba, Brazil. Arq. Ciên. Mar., v. 49, n 2, p. 92 - 103, 2016.

Nascimento, G.C.C.; Córdula, E.B.L.; Paiva, R.F.; Rosa, R.S. \& Lucena, J.S.M. Pescadores e "currais": um enfoque etnoecológico. Gaia Scient., v. 10, n. 4, p.117-137, 2016b.

Nottingham, M.C.; Cunha, F.E.A. \& Monteiro-Neto, C. Catching of ornamental marine fishes in Ceará State, Brazil. Arq. Ciên. Mar, Fortaleza, v. 33, p. 113-118, 2000.

Odum, E. P. Ecologia. 2. ed. Rio de Janeiro: Guanabara Koogan S.A., 2010. 434 p.

Paranaguá, M. N. Cladocera (Crustacea) do estuário do Rio Capibaribe - Recife - Pernambuco. 1991. 102 f. Tese (Professor Titular da Área de Zoologia) - Universidade Federal Rural de Pernambuco, Recife. 1991.

Pinheiro, I.E.G. \& Castello, J.P. Caracterização e comparação da abundância e diversidade de peixes recifais em diferentes enseadas da Ilha do Arvoredo: considerações sobre a "Reserva Biológica Marinha (REBIOMAR) do Arvoredo". Atlântica, Rio Grande, v. 32, n. 02, p. 127-140, 2010. http:/ / dx.doi.org/10.5088/atl.2010.32.2.127.

Ramires, M.; Clauzet, M.; Rotundo, M.M. \& Begossi, A. A pesca e os pescadores artesanais de Ilhabela (SP), Brasil. Bol. Inst. Pesca, São Paulo, v. 38, p. 2, p. 231 - 246, 2012.

Scolforo, J.R.; Oliveira, A.D.; Ferraz Filho, A.C. \& Mello, J.M. Diversidade, equabilidade e similaridade no domínio da caatinga. In: Mello, J.M.; Scolforo, J.R. \& Carvalho, L.M.T. (Ed.). Inventário Florestal de Minas Gerais: Floresta Estacional Decidual - Florística, Estrutura, Similaridade, Distribuição Diamétrica e de Altura, Volumetria, Tendências de Crescimento e Manejo Florestal. Lavras: UFLA, 2008. cap. 6, p.118-133.

Souza, L.A.; Freitas, C.E.C. \& Garcez, R.C.S. Relação entre guildas de peixes, ambientes e petrechos de pesca baseado no conhecimento tradicional de pescadores da Amazônia Central. Bol. Inst. Pesca, São Paulo, v. 41, n. 3, p. 633 - 644, 2015.

Viva Santos (2017). O Site de Cidade de Santos, Link Aquário de Santos. Disponível em: http:/ / www.vivasantos.com.br/aquario/atracoes/main.htm. Acessado em: 09 de junho de 2017. 